\title{
Analysis of Gene-Gene Interactions Using Gene-Trait Similarity Regression
}

\author{
Xin Wang ${ }^{a, b}$ Michael P. Epstein ${ }^{c} \quad$ Jung-Ying Tzeng ${ }^{a, b, d}$ \\ ${ }^{a}$ Bioinformatics Research Center, and b Department of Statistics, North Carolina State University, Raleigh, N.C., \\ and ${ }^{\mathrm{C}}$ Department of Human Genetics, Emory School of Medicine, Atlanta, Ga., USA; d Department of Statistics, \\ National Cheng-Kung University, Tainan, Taiwan, ROC
}

\section{Key Words}

Gene-gene interactions · Gene-trait similarity · Similarity regression

\begin{abstract}
Objective: Gene-gene interactions $(\mathrm{G} \times \mathrm{G})$ are important to study because of their extensiveness in biological systems and their potential in explaining missing heritability of complex traits. In this work, we propose a new similarity-based test to assess $G \times G$ at the gene level, which permits the study of epistasis at biologically functional units with amplified interaction signals. Methods: Under the framework of genetrait similarity regression (SimReg), we propose a genebased test for detecting $\mathrm{G} \times \mathrm{G}$. SimReg uses a regression model to correlate trait similarity with genotypic similarity across a gene. Unlike existing gene-level methods based on leading principal components (PCs), SimReg summarizes all information on genotypic variation within a gene and can be used to assess the joint/interactive effects of two genes as well as the effect of one gene conditional on another. Results: Using simulations and a real data application to the Warfarin study, we show that the SimReg GXG tests have satisfactory power and robustness under different genetic architecture when compared to existing gene-based interac-
\end{abstract}

tion tests such as PC analysis or partial least squares. A genome-wide association study with approx. 20,000 genes may be completed on a parallel computing system in 2 weeks.

(c) 2014 S. Karger AG, Basel

\section{Introduction}

Gene-gene interactions $(\mathrm{G} \times \mathrm{G})$ are important to study since they are believed to be widespread in biological systems $[1,2]$ and are likely involved in gene regulation, signal transduction, biochemical networks as well as other physiological and developmental pathways [3-5]. G×G can further provide insight into the missing heritability of complex traits [6-8] and explain replication failures of initial genome-wide association study (GWAS) findings $[9,10]$. Regarding this latter point, $G \times G$ help explain between-study differences in marginal genetic effects, which can be due to between-study differences in the frequency of modifier genetic variant(s). The study of $\mathrm{G} \times \mathrm{G}$ has provided insight into biological mechanisms for many complex diseases, including Alzheimer's disease, diabetes, cardiovascular disease, autism, multiple sclerosis, and cancer [11-17].

\section{KARGER}

E-Mail karger@karger.com

www.karger.com/hhe
(C) 2014 S. Karger AG, Basel

$0001-5652 / 14 / 0781-0017 \$ 39.50 / 0$
Jung-Ying Tzeng

Department of Statistics and Bioinformatics Research Center

Campus Box 7566, North Carolina State University

Raleigh, NC 27695-7566 (USA)

E-Mail jytzeng@stat.ncsu.edu 
Analysts often implement $G \times G$ tests using a regression model accounting for the main effects of two single nucleotide polymorphisms (SNPs) and also the twoway interaction between the two SNPs. The interaction effect can be assessed on the additive or the multiplicative scale - the former examines the effect on the phenotype on the linear scale, and the latter examines the effect on the log scale of the phenotype. The additive scale is often more relevant to public health importance [18], while the multiplicative scale more naturally corresponds to the biological mechanisms [19]. Regardless of the scale, one can test whether the interaction parameter is different from zero or, alternatively, consider a test similar to that of Chapman and Clayton [20] to assess the effect of a SNP in the presence of interaction with a second SNP. While analysts primarily applied such SNP-SNP interaction tests in small-scale candidate-gene studies, there has also been keen interest in performing exhaustive interaction testing of SNPs in a GWAS [21]. One can perform such genome-wide analyses using standard tools like regression [6]. However, one can also apply more innovative techniques such as two-stage screening procedures [22], Bayes networks [23], Bayesian model averaging [24], logic regression [25] and data-mining procedures [26-29].

All of these existing interaction methods consider the analysis on the level of a SNP. However, there is increasing interest in performing such analyses on the broader level of a gene [30-32]. Several factors motivate the paradigm shift from SNP to gene. First, genes are the basic unit in the biological mechanism and SNPs within a gene tend to work concordantly. Thus, gene-level results may be more biologically insightful and easier to interpret. Second, a gene-level analysis incorporates linkage disequilibrium (LD) information from all SNPs simultaneously within the gene. Consequently, such a joint analysis of SNPs should have an improved ability to tag untyped causal variants compared to the analysis of individual SNPs, leading to improved power. Finally, if a gene harbors multiple causal variants, then a joint analysis of SNPs in aggregate should be more powerful than a separate analysis of each individual SNP (owing in part to the gene-based test often having less degrees of freedom than its individual-SNP counterpart).

A few gene-based methods for interaction testing exist. Chatterjee et al. [33] proposed Tukey's 1-df method to investigate an interaction between two candidate genes. The approach calculates the sum of the main effects of SNPs contained within each gene and then uses the product of the two sums as the $\mathrm{G} \times \mathrm{G}$ term. Thus, the approach models one interaction parameter at the gene level rather than several interaction parameters on the SNP level. Owing to the reduced degrees of freedom of the Tukey test, the authors showed that their approach lead to a great improvement in power to detect $\mathrm{G} \times \mathrm{G}$ compared to individual-SNP analysis. Motivated by this idea, Wang et al. [32] considered two different interaction tests that summarized SNP information within a gene. For the first test, the authors used principal component analysis (PCA) to summarize LD information of SNPs within a gene. They then created an interaction test using the top (first) principal component (PC) from each gene. For their second test, the authors applied partial least squares (PLS) to extract components that summarize both the LD information among SNPs in a gene as well as the correlation between such SNPs and the outcome of interest. The authors then constructed their interaction test using the top PLS component from each gene. Using simulated data, the PCA and PLS methods often had better performance than the Tukey 1-df method, particularly when causal SNPs had no or a negligible marginal effect.

In this article, we propose a new gene-based test for detecting $G \times G$ in complex traits using similarity regression (SimReg) [34]. SimReg is an analytic procedure that uses a regression model to correlate trait similarity with genotypic similarity across a gene. SimReg is inspired by Haseman and Elston's regression from linkage analysis [35, 36] and haplotype similarity tests for regional association [37, 38]. In SimReg, the trait similarity is quantified by the trait covariance adjusting for the covariates. The multi-marker information of a gene is first summarized by genetic similarity, which is measured using a prespecified metric such as the proportion of alleles shared identical by state (IBS) across the gene. The $G \times G$ is then modeled by taking the product of the genetic similarities of the two genes, and its significance is assessed by testing the significance of the corresponding regression coefficient.

Compared to the Tukey-, PCA- or PLS-based methods, we believe SimReg has several advantages. First, SimReg utilizes all information within a gene, while the PCA or PLS procedures may lose some information due to considering only the top component of each gene within the interaction analysis. Second, as the top PCA or PLS component from each gene is a weighted sum of SNP information, the use of the product of the two linear combinations as the interaction term in the model only captures limited forms of non-additive effect. In contrast, SimReg provides a tool to model a variety of effects through different measures of summarized gene-similarity information. At the same time, SimReg does not involve a large number of parameters so that it has a good
18

Hum Hered 2014;78:17-26 DOI: $10.1159 / 000360161$
Wang/Epstein/Tzeng 
power performance. Finally, as we show later, we can obtain an analytic $p$ value for the SimReg procedure without the need for intensive resampling procedures like those required for the Tukey approach.

We arranged the remainder of the paper as follows. We first present the SimReg approach and describe how to use the framework to construct three score tests of interest (interaction test, joint test and conditional main effect test). Then, we derive the distributions of these tests under the null hypothesis by connecting the approach to a variance component model. We use simulated data to example the performance of the proposed method against the PCA and PLS methods as well as a standard SNPbased test of interaction. Later, we also apply SimReg to the Warfarin data from the study of Wysowski et al. [39]. Finally, we discuss further research directions of the proposed approach.

\section{Methods}

\section{The Gene-Trait Similarity Model}

We assume a sample of $N$ subjects. For subject $i(i=1, \ldots, N)$, we denote $Y_{i}$ as the trait value, $X_{i}$ as a $K \times 1$ vector of covariates (including the intercept term), and $G_{m, i}$ as a coding of the subject's genotype at SNP $m$. We define $S_{i j}^{A}$ to be the genetic similarity of gene A between subjects $i$ and $j(i \neq j)$. There are many ways to describe the genetic similarity between individuals. Here, we model similarity as the weighted sum of the proportion of alleles shared IBS across the $M_{A}$ SNPs in gene A. That is, for subjects $i$ and $j$, the similarity level is

$$
S_{i j}^{A}=\sum_{m=1}^{M_{A}} w_{m} s_{m, i j},
$$

where $s_{m, i j}=x / 2$, if $G_{m, i}$ and $G_{m, j}$ share $x$ alleles IBS [40, 41], and weight $w_{m}$ can be used to up-weight or down-weight a variant based on allele frequencies, the degree of evolutionary conservation or the functionality of the variations $[40,42,43]$. In this work, to up-weight similarities that are contributed by rare alleles, we set $w_{m}=q_{m}^{-1}$, where $q_{m}$ is the minor allele frequency (MAF) of marker $m[44,45]$. We can define the similarity level in gene B $S_{i j}^{B}$ in the same manner. The trait similarity between individuals $i$ and $j$, denoted by $Z_{i j}$, is computed by $Z_{i j}=\left(Y_{i}-\mu_{i}\right)\left(Y_{j}-\mu_{j}\right)$, where $\mu_{j}=$ $E\left(Y_{i} \mid X_{i}\right)=X_{i} \gamma$, which is the conditional mean of trait with no genotype effect, and where $\gamma$ is the effect of the covariates. The SimReg for $\mathrm{G} \times \mathrm{G}$ is

$$
E\left(Z_{i j} \mid X, G\right)=\tau_{A} S_{i j}^{A}+\tau_{B} S_{i j}^{B}+\tau_{A B} S_{i j}^{A} S_{i j}^{B} .
$$

Note that the regression model has zero intercept because we incorporated the covariate effects when quantifying trait similarity [34].

\section{The Interaction Test}

The interaction test examines the null hypothesis $H_{0, \text { Int }}: \tau_{A B}=0$. We derive the score test of this hypothesis from equation 1 by taking advantage of the connection between the similarity model and a variance component model [34]. Below, we first show that the regression coefficients in equation 1 can be viewed as the variance components under a mixed model. We then construct the test of $\tau_{A B}$ using the same test from the corresponding variance component model. Specifically, consider the following working mixed model

$$
Y_{i}=X_{i} \gamma+g_{A, i}+g_{B, i}+g_{A B, i}+e_{i},
$$

where $e_{i} \sim N(0, \sigma)$ and $g_{A, i}, g_{B, i}$ and $g_{A B, i}$ are subject-specified genetic effects for gene $A$, gene $B$ and $\mathrm{G} \times \mathrm{G}$, respectively. Let $g_{A}$ equal $\left[g_{A, 1}, \ldots, g_{A, n}\right], g_{B}$ equal $\left[g_{B, 1}, \ldots, g_{B, n}\right]$ and $g_{A B}$ equal $\left[g_{A B, 1}, \ldots, g_{A B, n}\right]$, and assume that $g_{A} \sim M N\left(0, v_{A} S_{A}\right), g_{B} \sim M N\left(0, v_{B} S_{B}\right)$ and $g_{A B} \sim$ $M N\left(0, v_{A B} S_{A B}\right)$, where $S_{A}=\left\{S_{i j}^{A}\right\}, S_{B}=\left\{S_{i j}^{B}\right\}$ and $S_{A B}=\left\{S_{i j}^{A} \times S_{i j}^{B}\right\}$. Under equation 2 , we can obtain the marginal trait covariance by

$$
\begin{aligned}
\operatorname{cov}\left(Y_{i}, Y_{j} \mid X, G\right)= & \operatorname{cov}_{g .}\left\{E\left(Y_{i} \mid \mathbf{X}, G, g_{A}, g_{B}, g_{A B}\right),\right. \\
& \left.E\left(Y_{i} \mid X, G, g_{A}, g_{B}, g_{A B}\right)\right\} \\
= & \operatorname{cov}_{g .}\left\{X_{i} \gamma+g_{A, i}+g_{B, i}+g_{A B, i}, X_{j} \gamma\right. \\
& \left.+g_{A, j}+g_{B, j}+g_{A B, j}\right\} \\
= & v_{A} S_{i j}^{A}+v_{B} S_{i j}^{B}+v_{A B} S_{i j}^{A} S_{i j}^{B} .
\end{aligned}
$$

Comparing equation 3 with equation 1 , we have $\tau_{A}=v_{A}, \tau_{B}=v_{B}$ and $\tau_{A B}=v_{A B}$.

We derive the score function of the restricted maximum likelihood (REML) log-likelihood function of equation 2 in Appendix A, and obtain the score statistic under $H_{0, \text { Int }}$ as

$$
T_{\text {Int }}=1 /\left.2 \mathbf{Y}^{\prime} P_{\text {Int }} S_{A B} P_{\text {Int }} \mathbf{Y}\right|_{\tau_{A}=\tau_{\hat{A}}, \tau_{B}=\tau_{\hat{B}}, \sigma=\hat{\sigma}},
$$

where $\left(Y_{1}, \ldots, Y_{n}\right), P_{I n t}=V_{I n t}^{-1}-V_{I n t}^{-1} \mathbf{X}\left(\mathbf{X}^{\prime-1} V_{I n t}^{-1} \mathbf{X}\right)^{-1} \mathbf{X}^{\prime} V_{\text {Int }}^{-1}$, $V_{\text {Int }}=\tau_{A} S_{A}+\tau_{B} S_{B}+\sigma I$ and $\left(\tau_{\hat{A}}, \tau_{\hat{B}}, \hat{\sigma}\right)$ are the maximum REML estimates obtained under $H_{0, \text { Int }}: \tau_{A B}=0$. We describe an expectationmaximization (EM) algorithm to obtain $\left(\tau_{\hat{A}}, \tau_{\hat{B}}, \hat{\sigma}\right)$ in Appendix B.

Under the alternative hypothesis $\tau_{A B} \neq 0, T_{\text {Int }}$ is a strictly increasing function of $\tau_{A B}$. Therefore larger values of $T_{\text {Int }}$ provide stronger evidence against $H_{0, \text { Int }}$. This suggests that the testing procedure should be one sided. As shown in Appendix A, the distribution of $T_{\text {Int }}$ follows a weighted $\chi^{2}$ distribution. That is, define

$$
C_{\text {Int }}=1 / 2 V^{1 / 2} P_{\text {Int }} S_{\text {In }} P_{\text {Int }} V_{\text {Int }}^{1 / 2},
$$

and then

$$
T_{\text {Int }} \sim \sum_{j=1}^{c} \lambda_{j, \text { Int }} \chi_{1}^{2},
$$

where $\lambda_{j, I n t}$ is the ordered nonzero eigenvalue of matrix $C_{\text {Int }}$. We can calculate the $\mathrm{p}$ values analytically using moment-matching approximations [46].

\section{The Joint Test}

Instead of performing a test specifically for interactions, one may be interested in assessing whether the two genes have any marginal or interactive effects on the trait [47]. This can be done using the joint test to examine $H_{0, \text { Int }}: \tau_{A}=\tau_{B}=\tau_{A B}=0$ under the full model $E\left(Z_{i j} \mid X, G\right)=\tau_{A} S_{i j}^{A}+\tau_{B} S_{i j}^{B}+\tau_{A B} S_{i j}^{A} S_{i j}^{B}$. As shown in Appendix $A$, the test statistic is given as

$$
T_{\text {Joint }}=1 /\left.2 \mathbf{Y}^{\prime} P_{\text {Joint }}\left(S_{A}+S_{B}+S_{A B}\right) P_{\text {Joint }} \mathbf{Y}\right|_{\sigma=\check{\sigma}} \text {, }
$$

where $P_{\text {Joint }}=\sigma^{-1}\left\{I-\mathbf{X}\left(\mathbf{X}^{\prime} \mathbf{X}\right)^{-1} \mathbf{X}^{\prime}\right\}$ and $\breve{\sigma}=\mathbf{Y}^{\prime}\left\{I-\mathbf{X}\left(\mathbf{X}^{\prime} \mathbf{X}\right)^{-1} \mathbf{X}^{\prime}\right\} \mathbf{Y} /$ $(n-K)$. The distribution of $T_{\text {Joint }}$ also follows a weighted $\chi^{2}$ distribution, i.e.

$$
T_{\text {Joint }} \sim \sum_{j=1}^{c} \lambda_{j, \text { Joint }} \chi_{1}^{2},
$$

with $\lambda_{j, \text { Joint }}$ being the ordered nonzero eigenvalue of $C_{\text {Joint }}=$ $1 / 2 V_{\text {Joint }}^{1 / 2} P_{\text {Joint }}\left(S_{A}+S_{B}+S_{A B}\right) P_{\text {Joint }} V_{\text {Joint. }}^{1 / 2}$ As with the interaction 
test, we calculate the $\mathrm{p}$ values of the joint test analytically using moment-matching procedures.

\section{The Conditional Main Effect Test}

We also construct the score test for the main effect of a gene conditioning on the effect of the other gene, assuming no $\mathrm{G} \times \mathrm{G}$. This is because in scenarios where the interaction effects do exist, the main effects are typically not well defined, and its significance depends on the scale of the interacting variables. When there is no $\mathrm{G} \times \mathrm{G}$, the full model is

$$
E\left(Z_{i j} \mid X, G\right)=\tau_{A} S_{i j}^{A}+\tau_{B} S_{i j}^{B}
$$

Here to test the main effect of Gene A accounting for the effect of Gene $\mathrm{B}$, we evaluate $H_{0, A}: \tau_{A}=0$. We can similarly test the main effect of Gene $B$ accounting for the effect of Gene A by evaluating $H_{0, B}: \tau_{B}=0$. Similar to the score tests in the interaction test and joint test, we derive the score test statistic for the conditional main effect of Gene A conditional on Gene B as

$$
T_{A}=1 /\left.2 \mathbf{Y}^{\prime} P_{A} S_{A} P_{A} \mathbf{Y}\right|_{\tau_{B}=\tilde{\tau}_{B}, \sigma=\tilde{\sigma}},
$$

where $P_{A}=V_{A}^{-1}-V_{A}^{-1} \mathbf{X}\left(\mathbf{X}^{\prime-1} V_{A}^{-1} \mathbf{X}\right)^{-1} \mathbf{X}^{\prime} V_{A}^{-1}, V_{A}=\tau_{B} S_{B}+\sigma I$ and $\left(\tilde{\tau}_{B}\right.$, $\tilde{\sigma})$ are the maximum REML estimates obtained under $H_{0, A}: \tau_{A}=0$. We describe the EM algorithms to obtain $\left(\tilde{\tau}_{B}, \tilde{\sigma}\right)$ in Appendix B. The test statistic $T_{B}$ can be defined similarity for examining $H_{0, B}$ : $\tau_{B}=0$ under the constrain of $\tau_{A B}=0$. As shown in Appendix $A, T_{A}$ has the same distribution as

$$
\sum_{j=1}^{c} \lambda_{j, A} \chi_{1}^{2},
$$

where $\lambda_{j, A}$ is the ordered nonzero eigenvalue of $C_{A}=1 / 2 V_{A}^{1 / 2}$ $P_{A} S_{A} P_{A} V_{A}^{1 / 2}$. As with the interaction and joint tests, we analytically derive the $\mathrm{p}$ values of the test statistic using moment-matching procedures.

\section{Simulation Study}

\section{Design}

We study the performance of the proposed methods using simulated data, and benchmark them against 3 approaches: (1) LR: linear regression; (2) PCA: the principal component method of Wang et al. [32], and (3) PLS: the partial least square of Wang et al. [32]. The LR incorporates all SNPs from each of the 2 genes as well as all pairwise interactions of SNPs across genes. When performing conditional main effect testing, we exclude the interaction terms from the LR analysis. We also only consider the proposed test and LR for the conditional main effect test, since PCA and PLS are identical to LR when there is no interaction term.

To simulate genotype data with realistic LD patterns, we use genotype data of gene RBJ (8 SNPs) and gene GPRC5B (15 SNPs) of the phase III CEU samples downloaded from the International HapMap Project (http://hapmap.ncbi.nlm.nih.gov/). We show the LD structure of the two genes in online supplementary figure 1 (see www.karger.com/doi/10.1159/000360161 for all online suppl. material) and the MAF and the average $R^{2}$ of each SNP in online supplementary table 1 . The average $R^{2}$ is calculated by averaging the $R^{2}$ between the target SNP and the remaining SNPs in the same gene. We consider two causal SNPs from each gene and simulate the trait values based on the model listed below.

\begin{tabular}{|c|c|c|c|c|c|c|c|c|}
\hline \multirow{2}{*}{$\begin{array}{l}\text { Sce- } \\
\text { nario }\end{array}$} & \multicolumn{2}{|l|}{$S N P_{1}^{A}$} & \multicolumn{2}{|l|}{$S N P_{2}^{A}$} & \multicolumn{2}{|l|}{$S N P_{1}^{B}$} & \multicolumn{2}{|l|}{$S N P_{2}^{B}$} \\
\hline & $\operatorname{avg} R^{2}$ & MAF & $\operatorname{avg} R^{2}$ & MAF & $\operatorname{avg} R^{2}$ & MAF & $\operatorname{avg} R^{2}$ & MAF \\
\hline 1 & 0.58 & 0.12 & 0.13 & 0.48 & 0.26 & 0.46 & 0.23 & 0.48 \\
\hline 2 & 0.57 & 0.12 & 0.16 & 0.06 & 0.14 & 0.16 & 0.23 & 0.48 \\
\hline 3 & 0.58 & 0.12 & 0.16 & 0.06 & 0.07 & 0.04 & 0.14 & 0.16 \\
\hline
\end{tabular}

Table 1. The causal SNPs for Gene A and Gene B for the three scenarios considered in the power analysis

$$
\begin{aligned}
& Y=\beta_{A} \times\left(S N P_{1}^{A}+S N P_{2}^{A}+S N P_{1}^{A} \times S N P_{2}^{A}\right) \\
& +\beta_{B} \times\left(S N P_{1}^{B}+S N P_{2}^{B}+S N P_{1}^{B} \times S N P_{2}^{B}\right) \\
& +\beta_{A B} \times\left(S N P_{1}^{A} \times S N P_{1}^{B}+S N P_{2}^{A} \times S N P_{2}^{B}\right)+e
\end{aligned}
$$

where $S N P_{1}^{A}$ and $S N P_{2}^{A}$ are the number of the minor alleles carried by a subject at the first and second causal loci in Gene A, $S N P_{1}^{B}$ and $S N P_{2}^{B}$ are defined similarly and $e$ follows a normally distributed variable with mean 0 and variance 1 . The trait value $Y$ is defined based on 3 parts: the gene effect from Gene A, the gene effect from Gene B and the interaction effect between Gene A and Gene B. We assume no LD between the genes RBJ and GPRC5B, and consequently sample the genotypes of the former gene independent of the genotypes for the latter gene.

To evaluate the type I error rate of the interaction test, we considered 3 situations of no $\mathrm{G} \times \mathrm{G}$ effects: (1) both genes have no genetic effect $\left(\beta_{A}=\beta_{B}=\beta_{A B}=0\right)$; (2) only Gene $\mathrm{A}$ has a main effect to the trait $\left(\beta_{A} \neq 0, \beta_{B}=\beta_{A B}=0\right)$, and (3) both Gene $A$ and Gene $\mathrm{B}$ have a main effect to the trait $\left(\beta_{A} \neq 0, \beta_{B} \neq 0, \beta_{A B}=0\right)$. To evaluate the type I error rate for the joint test, we set $\beta_{A}=\beta_{B}=\beta_{A B}=0$. To evaluate the type I error rate for the conditional main effect test, we examined the size strictly for Gene B $\left(H_{0}: \tau_{B}=0\right)$. We considered two scenarios of no effect of Gene B: (1) both Gene A and Gene $B$ have no main effect $\left(\beta_{A}=\beta_{B}=\beta_{A B}=0\right)$ and (2) Gene A has a main effect but not Gene $\mathrm{B}\left(\beta_{A} \neq 0, \beta_{B}=0, \beta_{A B}=0\right)$. For each scenario, we simulated datasets comprised of 300 subjects and evaluated the type I error rates using 1,000 replicates of the data.

For power analysis, we consider three different scenarios based on the causal SNPs. Specifically, we pick up 'representative' SNPs from all SNPs with similar LD and MAF patterns and form 3 different causal SNP combinations (table 1). When generating trait values using the model above, we consider different $\beta$ values so that the power of the different methods at a significance threshold of $5 \%$ is between 20 and $80 \%$. The power was calculated based on 200 replications.

\section{Results}

Table 2 shows the empirical type I error rates for all methods at a significance level of $\alpha=0.05$. The type I error rates of all approaches are around the nominal level in all different settings. For the interaction test, the type I error rates of the proposed method are slightly conservative but we observed that, as the variance due to the main effects 
Table 2. The type I error rate at significance level 0.05 for the three tests

\begin{tabular}{|c|c|c|c|c|c|c|}
\hline \multirow[t]{2}{*}{ Method } & \multicolumn{3}{|c|}{ Interaction test } & \multirow{2}{*}{$\begin{array}{l}\text { Joint test } \\
(0,0,0)\end{array}$} & \multicolumn{2}{|c|}{$\begin{array}{l}\text { Conditional main effect } \\
\text { test }(\mathrm{B} \mid \mathrm{A})\end{array}$} \\
\hline & $(0,0,0)$ & $(0.1,0,0)$ & $(0.1,0.1,0)$ & & $(0,0,0)$ & $(0.3,0,0)$ \\
\hline SimReg & 0.038 & 0.04 & 0.046 & 0.049 & 0.048 & 0.052 \\
\hline LR & 0.060 & 0.062 & 0.064 & 0.060 & 0.060 & 0.056 \\
\hline PCA & 0.056 & 0.06 & 0.058 & 0.055 & & \\
\hline PLS & 0.046 & 0.048 & 0.049 & 0.054 & & \\
\hline
\end{tabular}

$\left(\beta_{\mathrm{A}}, \beta_{\mathrm{B}}, \beta_{\mathrm{AB}}\right)$ is the effect size.

Table 3. Power analysis for different approaches at significance level 0.05 under the scenarios listed in table 1

\begin{tabular}{lllll}
\hline & SimReg & LR & PCA & PLS \\
\hline Interaction test & & & & \\
Scenario 1 $(0.1,0.1,0.3)$ & $\mathbf{0 . 6 9 0}(0.626,0.754)^{\mathrm{a}}$ & $0.375(0.308,0.442)$ & $\mathbf{0 . 7 1 5}(0.652,0.778)$ & $\mathbf{0 . 7 4 5}(0.685,0.805)$ \\
Scenario 2 $(0.1,0.1,0.5)$ & $\mathbf{0 . 4 6 0}(0.391,0.529)$ & $0.285(0.222,0.348)$ & $\mathbf{0 . 3 5 0}(0.284,0.416)$ & $\mathbf{0 . 4 9 0}(0.421,0.559)$ \\
Scenario 3 $(0.1,0.1,1.5)$ & $\mathbf{0 . 8 1 5}(0.761,0.869)$ & $\mathbf{0 . 7 6 0}(0.701,0.819)$ & $0.120(0.075,0.165)$ & $0.470(0.401,0.539)$ \\
\hline Joint test & & & & $0.535(0.466,0.604)$ \\
Scenario 1 $(0.05,0.05,0.05)$ & $\mathbf{0 . 6 7 0}(0.605,0.735)^{\mathrm{a}}$ & $0.325(0.260,0.390)$ & $\mathbf{0 . 5 4 5}(0.476,0.614)$ \\
Scenario 2 $(0.1,0.1,0.1)$ & $\mathbf{0 . 8 7 5}(0.829,0.921)$ & $0.690(0.626,0.754)$ & $0.765(0.706,0.824)$ & $\mathbf{0 . 7 8 5}(0.728,0.842)$ \\
Scenario 3 $(0.3,0.3,0.3)$ & $\mathbf{0 . 5 8 0}(0.512,0.648)$ & $0.325(0.260,0.390)$ & $\mathbf{0 . 5 0 0}(0.431,0.569)$ & $\mathbf{0 . 5 0 5}(0.436,0.574)$ \\
\hline Conditional main effect test & & & & \\
Scenario 1 $(0.07,0.07,0)$ & $\mathbf{0 . 6 5 0}(0.584,0.716)^{\mathrm{a}}$ & $0.415(0.347,0.483)$ & & \\
Scenario 2 $(0.1,0.1,0)$ & $\mathbf{0 . 6 8 5}(0.621,0.749)$ & $0.475(0.406,0.544)$ & & \\
Scenario 3 $(0.3,0.3,0)$ & $\mathbf{0 . 4 9 5}(0.426,0.564)$ & $\mathbf{0 . 4 8 5}(0.416,0.554)$ & & \\
\hline
\end{tabular}

In each scenario, the effect size $\left(\beta_{A}, \beta_{B}, \beta_{A B}\right)$ is set so that the power range is between 20 and $80 \%$. The values in italics and bold indicate the method with the largest power under a certain scenario; the values in bold indicate the methods with overlapping confidence intervals with the best methods.

a $95 \%$ confidence interval of the power, obtained by $\hat{p} \pm 1.96 \times \sqrt{\hat{p} \times(1-\hat{p}) / 200}$, with $\hat{p}$ being the empirical power estimated based on 200 replications.

of Gene A and/or Gene B increases, the type I error rate generally approaches the nominal level. As discussed in the Appendix, the conservative nature of the interaction test arises because of the bias in the EM algorithm estimates of the variance components when their true values are 0 . The type I error rates for the interaction test using the competing methods (PCA, PLS, LR) are generally appropriate. For joint and conditional main effect tests, we observed the type I error rates of all methods considered in our analyses to be quite similar to the nominal levels.

We next performed a power analysis under the models considered in table 1. Because different scenarios have different causal SNP combinations, we adjust the different $\beta$ values so that the power of the different methods is around $0.2-0.8$. The results are summarized in table 3 .
Overall, we observed that the proposed method generally has optimal power relative to the other methods considered under various genetic architectures of the causal SNPs. For the interaction test, the causal SNPs have relatively high LD and large MAFs in Scenario 1. PCA and PLS perform the best, closely followed by the proposed method. LR has the least power due to the large degree of freedom used. In Scenario 2, where the causal SNPs have a relatively smaller LD and MAF, PLS still has the best power, closely followed by the proposed method. The power of PCA drops a lot in this scenario. LR again has the least power. In Scenario 3, the proposed method has the best power, followed by LR, PLS and PCA. PCA and PLS have a significantly low power performance in Scenario 3. As the LD and MAF become smaller, the 
Table 4. $\mathrm{p}$ values of the 4 approaches in the warfarin analysis

\begin{tabular}{lllll}
\hline Method & Joint test & \multicolumn{2}{l}{$\begin{array}{l}\text { Interaction } \\
\text { test }\end{array}$} & Conditional main effect test \\
\cline { 3 - 5 } & & & VKORC1 & CYP2C9 \\
\hline SimReg & $5.6 \times 10^{-20}$ & 0.450 & $1.67 \times 10^{-20}$ & $1.02 \times 10^{-7}$ \\
LR & $1.92 \times 10^{-16}$ & 0.753 & $1.94 \times 10^{-16}$ & $2.65 \times 10^{-8}$ \\
PCA & $8.07 \times 10^{-5}$ & 0.530 & - & - \\
PLS & $7.43 \times 10^{-5}$ & 0.470 & - & - \\
\hline
\end{tabular}

power of PCA drops dramatically, because the first PC can only capture a limited amount of information on the causal SNPs. The first PC aims to maximize the SNP variation captured and tends to be dominated by SNPs with high LD or common MAFs, which are non-causal SNPs in Scenario 3. PLS shares the same issue as PCA and hence it also suffers from power loss when the LD and MAFs of the causal SNPs become smaller. However, PLS also accounts for the trait information, which makes PLS perform better than PCA. The power advantage of PLS over PCA is more substantial in Scenarios 2 and 3, where an increased proportion of causal SNPs have low MAFs. Consequently, the first PC of PCA (which explains the largest variance among the genotypes) is less likely to harbor such causal SNPs and therefore PCA has a reduced power for interaction testing compared to PLS [32].

For the joint test, under the three different scenarios, the proposed method performs the best. PCA and PLS perform similarly with a slightly better power of PLS. The observation is consistent with the results of Wang et al. [32], because the first PC from PLS considers LD and a correlation between trait and gene. LR has the lowest power, which may be due to the large degree of freedom used in the joint test.

For the conditional main effect test, the proposed method always has a better power than linear models, but the difference between the proposed method and the linear models becomes smaller when the LD and MAFs of the causal SNPs become smaller. The finding is consistent with the finding in the interaction test.

\section{Real Data Analysis}

Warfarin is a widely used oral anticoagulant. In 2004, more than 30 million prescriptions contained this drug in the USA [39]. The optimal dose of warfarin is different from patient to patient, and an inappropriate dosage can lead to severe consequences such as bleeding and swelling of face or throat. Extensive research has been conducted to develop methods for predicting the appropriate dose.

We have conducted a genetic analysis using the data from the Warfarin study [48]. In this dataset, we studied the relationship between a stable warfarin dose and two genes: VKORC1 (containing 7 SNPs) and CYP2C9 (a triallelic locus). We further adjusted for 4 covariates associated with warfarin therapy: age, sex, height, and weight. After quality control, the dataset consisted of 301 individuals. We applied the proposed method and the benchmark methods to evaluate the association between warfarin dose and the two genes, adjusting for covariates. The results are summarized in table 4 . All methods identified a significant association between warfarin dose and the two genes. The smallest $\mathrm{p}$ value of the joint test was obtained from SimReg (i.e. $p=5.6 \times 10^{-20}$ ), closely followed by LR (i.e. $p=1.92 \times 10^{-16}$ ). The $p$ values from PCA and PLS are similar to each other and are many orders of magnitude larger (i.e. $10^{-5}$ ) than those derived from SimReg and LR. We next examined the interaction effect between VKORC1 and CYP2C9 and observed no significant interaction using any of the methods considered. Finally, we performed the conditional main effect test for each gene using SimReg and LR. The result suggests that both genes had significant effects on warfarin dose, with VKORC1 demonstrating a stronger effect (i.e. $p$ values on the order of $10^{-16}-10^{-20}$ ) than CYP2C9 (i.e. $p$ values on the order of $\left.10^{-7}-10^{-8}\right)$.

The main effect results were consistent with those in the current literature [49-51]. Previous studies indicate contradictory results regarding the interaction between VKORC1 and CYP2C9 on anticoagulant effect and hence the dose requirement [52-56]. Our results agree with recent reports $[55,56]$, which suggest no evidence of $G \times G$ between CYP2C9 and VKORC1.

\section{Discussion}

In this article, we describe a novel similarity-based test to assess $\mathrm{G} \times \mathrm{G}$ in large-scale association studies. Unlike the majority of existing procedures in this area, our approach considers the analysis at the level of the gene rather than of the SNP, which we show can lead to considerable improvements in power as a result. We also compare the performance of our similarity approach to existing gene-based interaction tests (PCA and PLS) and show that our joint, interaction and conditional mean effect tests nearly always had optimal power across the simula-
Wang/Epstein/Tzeng 
tion models. Relative to the PCA and PLS approaches, we believe our approach can improve power since it summarizes all information on genotypic variation within a gene. PCA and PLS, on the other hand, only use a subset of this variation within their analytic frameworks. The $\mathrm{R}$ code that implements the SimReg testing procedure is available from the authors' website (http://www4.stat.ncsu. edu/ jytzeng/Software/SimReg-GxG-QT/). Using a personal computer equipped with Intel Core i7-3770, 40 $\mathrm{GHz}$ CPU and $20 \mathrm{~GB}$ RAM, 200 runs of the interaction test (which is the slowest test because it requires estimating two nuisance variance components) with the simulated datasets required $767 \mathrm{~s}$. While the approach is not scalable to genome-wide interaction testing using a single processor, we believe the procedure can be applied to genome-wide data in reasonable time using a parallel-computing cluster that possesses a large number $(1,000)$ of CPUs.

In the SimReg model, we define the similarity function for two subjects as the weighted average of SNP alleles shared IBS by the pair across a gene. The weights are SNP specific and user defined. It is common to weight SNPs based on MAF such that rarer variants are assigned more weight than common variants; common weights in this setting include the reciprocal of a SNP's MAF or its square root [40,41]. Additionally, we can weight SNPs using functional information; perhaps providing more weight to SNPs that demonstrate evidence of being a cis-acting expression quantitative trait locus [57]. While we considered only common SNP variants in this work, we note that our SimReg approach can also integrate information from rare variants ascertained using next-generation sequencing technology. While weighting rare variants by MAF has obvious value, additional weighting of such variants using some measure that predicts the probability that the variant is deleterious and lowers fitness likely has value as well. A variety of computational algorithms exists for such predictions based on evolutionary, biochemical and/or structural information [for an overview, see 58].

We applied our similarity approach in the context of a candidate-gene study, but we can further expand the approach to consider interactions on a genome-wide scale. A gene-based interaction test has appealing features over SNP-based interaction tests when applied to a GWAS. Given that the number of genes is many-fold smaller than the typical number of SNPs in a GWAS, the number of tests that need to be evaluated using SimReg would be substantially smaller than a SNP-based interaction test like LR. This could substantially reduce the computational burden. Additionally, the issue of adjusting for multiple testing when conducting genome-wide interaction testing of SNPs is challenging. Within GWAS, most studies adjust for multiple testing using permutations, since a Bonferroni correction leads to conservative inference due to LD among SNPs. However, while permutations are valid for multiple testing adjustment in main effect tests, they are not valid when applied to interaction tests because such random shuffling of phenotypes does not preserve the observed main effects in the sample [59]. In contrast, multiple testing adjustment of interaction tests based on SimReg is more straightforward. While the application of gene-based SimReg to GWAS data will lead to correlated tests (due to repeated testing of the same gene) such that a Bonferroni correction is inappropriate, we can adjust for multiple testing using a computationally efficient perturbation procedure similar to that proposed by $\mathrm{Wu}$ et al. [60] that preserves the main effects of the genes under consideration. We will explore this work in a future paper.

\section{Appendix A}

Derivation of the Score Tests and Their Distributions Consider the matrix presentation of equation 2

$\mathbf{Y}=\mathbf{X} \gamma+g_{A}+g_{B}+g_{A B}+e$.

The corresponding REML log-likelihood function, denoted as $L\left(\tau_{A}, \tau_{B}, \tau_{A B}, \sigma\right)$, is

$$
L(\theta)=-1 / 2\left[\log |V|+\log \left|\mathbf{X}^{\prime-1} V \mathbf{X}\right|+\mathbf{Y}^{\prime} P \mathbf{Y}\right],
$$

where $V=\operatorname{Var}(Y)=\tau_{A} S_{A}+\tau_{B} S_{B}+\tau_{A B} S_{A B}+\sigma I$ is the marginal variance of $\mathbf{Y}$ and $P=V^{-1}-V^{-1} \mathbf{X}\left(\mathbf{X}^{\prime-1} V^{-1} \mathbf{X}\right)^{-1} \mathbf{X}^{\prime} V^{-1}$ is the projection matrix for the model. The score functions of $\tau_{A}, \tau_{B}$, and $\tau_{A B}$ based on $L(\theta)$ are

$$
\begin{aligned}
U_{\tau_{A}}\left(\tau_{A}, \tau_{B}, \tau_{A B}, \sigma\right) & =\frac{\partial L\left(\tau_{A}, \tau_{B}, \tau_{A B}, \sigma\right)}{\partial \tau_{A}} \\
& =\frac{1}{2}\left[\mathbf{Y}^{\prime} P S_{A} P \mathbf{Y}-\operatorname{tr}\left(P S_{A}\right)\right], \\
U_{\tau_{B}}\left(\tau_{A}, \tau_{B}, \tau_{A B}, \sigma\right) & =\frac{\partial L\left(\tau_{A}, \tau_{B}, \tau_{A B}, \sigma\right)}{\partial \tau_{B}} \\
& =\frac{1}{2}\left[\mathbf{Y}^{\prime} P S_{B} P \mathbf{Y}-\operatorname{tr}\left(P S_{B}\right)\right],
\end{aligned}
$$

and

$$
\begin{aligned}
U_{\tau_{A B}}\left(\tau_{A}, \tau_{B}, \tau_{A B}, \sigma\right) & =\frac{\partial L\left(\tau_{A}, \tau_{B}, \tau_{A B}, \sigma\right)}{\partial \tau_{A B}} \\
& =\frac{1}{2}\left[\mathbf{Y}^{\prime} P S_{A B} P \mathbf{Y}-\operatorname{tr}\left(P S_{A B}\right)\right] .
\end{aligned}
$$

We construct the statistics based on the first terms of the score functions. Here after, we define matrices $P_{h}$ and $V_{h}$ as $P$ and $V$ 
evaluated under $H_{0, h}$. Then for the interaction test $H_{0, \text { Int }}: \tau_{A B}=0$, we set the test statistic as

$$
T_{\text {Int }}=1 /\left.2 \mathbf{Y}^{\prime} P_{\text {Int }} S_{A B} P_{\text {Int }} \mathbf{Y}\right|_{\tau_{A}=\hat{\tau}_{A}, \tau_{B}, \sigma=\hat{\sigma}},
$$

where $P_{\text {Int }}=V_{\text {Int }}^{-1}-V_{\text {Int }}^{-1} \mathbf{X}\left(\mathbf{X}^{\prime-1} V_{\text {Int }}^{-1} \mathbf{X}\right)^{-1} \mathbf{X}^{\prime} V_{\text {Int }}^{-1}, V_{\text {Int }}=\tau_{A} S_{A}+\tau_{B} S_{B}$ $+\sigma I$ and $\left(\hat{\tau}_{A}, \hat{\tau}_{B}, \hat{\sigma}\right)$ are the maximum REML estimates obtained under $H_{0, \text { Int }}: \tau_{A B}=0$.

For the conditional main effect test $H_{0, A}: \tau_{A}=0$ under the constrain of no interaction (i.e. $\tau_{A B}=0$ ), we set the test statistic as

$$
T_{A}=1 /\left.2 \mathbf{Y}^{\prime} P_{A} S_{A} P_{A} \mathbf{Y}\right|_{\tau_{B}=\tilde{\tau}_{B}, \sigma=\tilde{\sigma}},
$$

where $P_{A}=V_{A}^{-1}-V_{A}^{-1} \mathbf{X}\left(\mathbf{X}^{\prime-1} V_{A}^{-1} \mathbf{X}\right)^{-1} \mathbf{X}^{\prime} V_{A}^{-1}, V_{A}=\tau_{B} S_{B}+\sigma I$ and $\left(\tilde{\tau}_{B}\right.$, $\tilde{\sigma})$ are the maximum REML estimates obtained under $H_{0, A}: \tau_{A}=0$. The test statistic $T_{B}$ can be defined similarly for examining $H_{0, B}: \tau_{B}=0$ under the constrain of $\tau_{A B}=0$. We describe the EM algorithms that we use to obtain $\left(\hat{\tau}_{A}, \hat{\tau}_{B}, \hat{\sigma}\right)$ and $\left(\tilde{\tau}_{B}, \tilde{\sigma}\right)$ in Appen$\operatorname{dix} B$.

For the joint test $H_{0, \text { Joint }}: \tau_{A}=\tau_{B}=\tau_{A B}=0$, because the values of $\tau$ are non-negative variance components, $\tau_{A}=\tau_{B}=\tau_{A B}=0$ if and only if $\tau_{A}+\tau_{B}+\tau_{A B}=0$. This motivates us to construct the test statistic based on the sum of the three score functions. That is

$$
T_{\text {Joint }}=1 / 2 \mathbf{Y}^{\prime} P_{\text {Joint }}\left(S_{A}+S_{B}+S_{A B}\right) P_{\text {Joint }} \mathbf{Y} I_{\sigma=\check{\sigma}} \text {, }
$$

where the $P_{\text {Joint }}=V_{\text {Joint }}^{-1}-V_{\text {Joint }}^{-1} \mathbf{X}\left(\mathbf{X}^{\prime-1} V_{\text {Joint }}^{-1} \mathbf{X}\right)^{-1} \mathbf{X}^{\prime} V_{\text {Joint }}^{-1}, V_{\text {Joint }}=$ $\sigma I$ and $\breve{\sigma}=\mathbf{Y}^{\prime}\left\{I-\mathbf{X}\left(\mathbf{X}^{\prime} \mathbf{X}\right)^{-1} \mathbf{X}^{\prime}\right\} \mathbf{Y} /(n-K)$.

The distributions of the test statistics can be shown to follow a weighted $\chi^{2}$ distribution via the fact that these statistics are a quadratic form of $\mathbf{Y}$. To illustrate this, consider $T_{\text {Int }}=1 / 2 \mathbf{Y}^{\prime} P_{\text {Int }} S_{A B}$ $P_{\text {Int }} \mathbf{Y}$. As $P_{\text {Int }}$ is a projection matrix, $P_{\text {Int }} \mathbf{X} \gamma=0$. Therefore,

$$
\begin{aligned}
T_{\text {Int }} & =\frac{1}{2} \mathbf{Y}^{\prime} P_{\text {Int }} S_{A B} P_{\text {Int }} \mathbf{Y}=\frac{1}{2}(\mathbf{Y}-\mathbf{X} \gamma)^{\prime} P_{\text {Int }} S_{A B} P_{\text {Int }}(\mathbf{Y}-\mathbf{X} \gamma) \\
& =\frac{1}{2}(\mathbf{Y}-\mathbf{X} \gamma)^{\prime} V_{\text {Int }}^{-1 / 2} V_{\text {Int }}^{1 / 2} P_{\text {Int }} S_{A B} P_{\text {Int }} V_{\text {Int }}^{1 / 2} V_{\text {Int }}^{-1 / 2}(\mathbf{Y}-\mathbf{X} \gamma) \sim \sum_{i=1}^{c} \lambda_{i} \chi_{1}^{2} .
\end{aligned}
$$

Define $\mathbf{z}=V^{-1 / 2}(\mathbf{I n t}-\mathbf{X} \gamma)$ and $C_{\text {Int }}=1 / 2 V^{-1 / 2}{ }_{\text {Int }} P_{\text {Int }} S_{A B} P_{\text {Int }} V_{\text {Int }}^{-1 / 2}$, we have

$$
T_{\text {Int }}=\mathbf{z}^{\prime} C_{\text {Int }} \mathbf{z} \sim \sum_{j=1}^{c} \lambda_{j, \text { Int }} \chi_{1}^{2},
$$

where $\lambda_{j, \text { Int }}$ is the ordered nonzero eigenvalue of matrix $C_{I n t}$. By the same manner, one can obtain that

$$
T_{A} \sim \sum_{j=1}^{c} \lambda_{j, A} \chi_{1}^{2},
$$

with $\lambda_{j, A}$ being the ordered nonzero eigenvalue of $C_{A}=$ $1 / 2 V_{A}^{1 / 2} P_{A} S_{A} P_{A} V^{1 / 2}$, and

$$
T_{\text {Joint }} \sim \sum_{j=1}^{c} \lambda_{j, \text { Joint }} \chi_{1}^{2} \text {, }
$$

with $\lambda_{j, \text { Joint }}$ being the ordered nonzero eigenvalue of $C_{\text {Joint }}=$ $1 / 2 V^{-1 / 2}$ Joint $P_{\text {Joint }}\left(S_{A}+S_{B}+S_{A B}\right) P_{\text {Joint }} V^{-1 / 2}$ Joint .

\section{Appendix B}

The EM Algorithm to Obtain the Maximum REML Estimates

We first describe the EM algorithm for $\left(\hat{\tau}_{A}, \hat{\tau}_{B}, \hat{\sigma}\right)$, i.e. the maximum REML estimates under $H_{0, \text { Int }}: \tau_{A B}=0$. Under $H_{0, \text { Int }}$, the LMM is

$$
\mathbf{Y}=\mathbf{X} \gamma+g_{A}+g_{B}+e,
$$

where $e \sim N(0, \sigma I), g_{A} \sim N\left(0, \tau_{A} S_{A}\right)$ and $g_{B} \sim N\left(0, \tau_{B} S_{B}\right)$ as $\tau_{A}=$ $v_{A}$ and $\tau_{B}=v_{B}$. Define $U=A^{T} \mathbf{Y}$ with the restriction that $A^{T} A=$ $I_{n-K}$ and $A A^{T}=I-\mathbf{X}\left(\mathbf{X}^{\prime} \mathbf{X}\right)^{-1} \mathbf{X}$. Then $U \mid g_{A}, g_{B} \sim N\left(A^{\prime} g_{A}+A^{\prime} g_{B}\right.$, $\left.\sigma I_{n-K}\right)$, which is independent of the fixed effect $\hat{\gamma}=\left(X^{T} X\right)^{-1} X^{T} Y$. Therefore the maximum REML estimates can be obtained by maximizing the marginal distribution of $U$, i.e. $f(U)=\int f\left(U \mid g_{A}, g_{B}\right)$ $f\left(g_{A}\right) f\left(g_{B}\right) d g_{A} d g_{B}$. This motivated an EM algorithm based on $U$ (i.e. the observed data) and $\left(g_{A}, g_{B}\right)$ (i.e. the missing data). The complete data log-likelihood, based on $f\left(U, g_{A}, g_{B}\right)$, is

$$
\begin{aligned}
\log f\left(U, g_{A}, g_{B} ; \tau_{A}, \tau_{B}, \sigma\right) \\
=\log f\left(U \mid g_{A}, g_{B} ; \tau_{A}, \tau_{B}, \sigma\right)+\log f\left(g_{A} ; \tau_{A}, \tau_{B}, \sigma\right)+\log f\left(g_{B} ; \tau_{A}, \tau_{B}, \sigma\right) \\
=-\frac{n-K}{2} \log \sigma-\frac{1}{2 \sigma}\left(U-A^{\prime} g_{A}-A^{\prime} g_{B}\right)^{\prime}\left(U-A^{\prime} g_{A}-A^{\prime} g_{B}\right) \\
\quad-\frac{q_{A}}{2} \log \tau_{A}-\frac{1}{2} \log \left(\left|S_{A}\right|_{+}\right)-\frac{1}{2 \tau_{A}} g_{A}^{T} S_{A}^{-} g_{A} \\
\quad-\frac{q_{B}}{2} \log \tau_{B}-\frac{1}{2} \log \left(\left|S_{B}\right|_{+}\right)-\frac{1}{2 \tau_{B}} g_{B}^{T} S_{B}^{-} g_{B},
\end{aligned}
$$

where $q_{A}$ and $q_{B}$ are the rank for matrix $S_{A}$ and $S_{B}$, respectively, $\left|S_{A}\right|_{+}$is the pseudodeterminant and $S_{A}^{-}$is the generalized inverse (as $S_{A}$ and $S_{B}$ may be singular).

In the expectation step, we compute

$Q\left(\tau_{A}, \tau_{B}, \sigma ; \hat{\tau}_{A}^{(t)}, \hat{\tau}_{B}^{(t)}, \hat{\sigma}^{(t)}\right) \equiv E\left\{\log f\left(U, g_{A}, g_{B} ; \tau_{A}, \tau_{B}, \sigma\right) \mid U ; \hat{\tau}_{A}^{(t)}, \hat{\tau}_{B}^{(t)}, \hat{\sigma}^{(t)}\right\}$

$=-\frac{n-K}{2} \log \sigma-\frac{1}{2 \sigma} E\left\{\left(U-A^{\prime} g_{A}-A^{\prime} g_{B}\right)^{\prime}\left(U-A^{\prime} g_{A}-A^{\prime} g_{B}\right) \mid U ; \hat{\tau}_{A}^{(t)}, \hat{\tau}_{B}^{(t)}, \hat{\sigma}^{(t)}\right\}$

$-\frac{q_{A}}{2} \log \tau_{A}-\frac{1}{2} \log \left(\left|S_{A}\right|_{+}\right)-\frac{1}{2 \tau_{A}} E\left(g_{A}^{T} S_{A}^{-} g_{A} \mid U ; \hat{\tau}_{A}^{(t)}, \hat{\tau}_{B}^{(t)}, \hat{\sigma}^{(t)}\right)$

$-\frac{q_{B}}{2} \log \tau_{B}-\frac{1}{2} \log \left(\left|S_{B}\right|_{+}\right)-\frac{1}{2 \tau_{B}} E\left(g_{B}^{T} S_{B}^{-} g_{B} \mid U ; \hat{\tau}_{A}^{(t)}, \hat{\tau}_{B}^{(t)}, \hat{\sigma}^{(t)}\right)$.

In the maximization step, we solve for $\partial Q / \partial \tau_{A}=0, \partial Q / \partial \tau_{B}=0$ and $\partial Q / \partial \sigma=0$, and obtain

$\hat{\tau}_{A}=\frac{1}{q_{A}} E\left(g_{A}^{T} S_{A}^{-} g_{A} \mid U ; \hat{\tau}_{A}^{(t)}, \hat{\tau}_{B}^{(t)}, \hat{\sigma}^{(t)}\right)=\frac{1}{q_{A}}\left\{\tilde{\mathbf{g}}_{A}^{(t)} S_{A}^{-} \tilde{\mathbf{g}}_{A}^{(t)}+\operatorname{tr}\left(S_{A}^{-} \tilde{\mathbf{v}}_{A}^{(t)}\right)\right\}$,

where

$\tilde{\mathbf{g}}_{A}^{(t)} \equiv E\left(g_{A} \mid g_{B}, U ; \hat{\tau}_{A}^{(t)}, \hat{\tau}_{B}^{(t)}, \hat{\sigma}^{(t)}\right)=\left.\tau_{A} S_{A} P_{I n t} Y\right|_{\hat{\tau}_{A}^{(t)}, \hat{\tau}_{B}^{(t)}, \hat{\sigma}^{(t)}}$,

and

$\tilde{\mathbf{v}}_{A}^{(t)} \equiv \operatorname{Var}\left(g_{A} \mid g_{B}, U ; \hat{\tau}_{A}^{(t)}, \hat{\tau}_{B}^{(t)}, \hat{\sigma}^{(t)}\right)=\tau_{A} S_{A}-\left.\tau_{A}^{2} S_{A} P_{I n t} S_{A}\right|_{\hat{\tau}_{A}^{(t)}, \hat{\tau}_{B}^{(t)}, \hat{\sigma}^{(t)}}$.

Similary, we have

$\hat{\tau}_{B}=\frac{1}{q_{B}} E\left(g_{B}^{T} S_{B}^{-} g_{B} \mid U ; \hat{\tau}_{A}^{(t)}, \hat{\tau}_{B}^{(t)}, \hat{\sigma}^{(t)}\right)=\frac{1}{q_{B}}\left\{\tilde{\mathbf{g}}_{B}^{(t) \prime} S_{B}^{-} \tilde{\mathbf{g}}_{B}^{(t)}+\operatorname{tr}\left(S_{B}^{-} \tilde{\mathbf{v}}_{B}^{(t)}\right)\right\}$.

Finally,

$$
\begin{aligned}
& \hat{\sigma}^{(t)}=\frac{1}{n-K} E\left\{\left(U-A^{\prime} g_{A}-A^{\prime} g_{B}\right)^{\prime}\left(U-A^{\prime} g_{A}-A^{\prime} g_{B}\right) \mid U ; \hat{\tau}_{A}^{(t)}, \hat{\tau}_{B}^{(t)}, \hat{\sigma}^{(t)}\right\} \\
& =Y^{* T} A A^{\prime} Y^{*}+\operatorname{tr}\left[A A^{\prime}\left(\begin{array}{l}
\hat{\tau}_{A}^{(t)} S_{A}-\left(\hat{\tau}_{A}^{(t)}\right)^{2} S_{A} P S_{A}+\hat{\tau}_{B}^{(t)} S_{B}-\left(\hat{\tau}_{B}^{(t)}\right)^{2} S_{B} P S_{B} \\
-2 \hat{\tau}_{A}^{(t)} \hat{\tau}_{B}^{(t)} S_{A} P S_{B}
\end{array}\right)\right] .
\end{aligned}
$$

The EM algorithm for obtaining $\left(\tilde{\tau}_{B}, \tilde{\sigma}\right)$ under $H_{0, A}: \tau_{A}=0$ is similar to the above algorithm except that $\tau_{A}$ is set to be 0 .

When applying the above EM algorithm, we add an additional testing step as detailed below. As the EM algorithm provides non- 
negative estimates, when $\tau_{A}$ and $\tau_{B}$ are 0 or close to 0 , the EM estimates obtained from the above algorithm can be biased as

$$
E\left(\widehat{\tau_{A}}\right)>\tau_{A}
$$

and

$$
E\left(\widehat{\tau_{B}}\right)>\tau_{B} .
$$

To solve this problem, we first apply conditional main effect tests for $H_{0}: \tau_{A}=0$ and $H_{0}: \tau_{B}=0$ to examine if these nuisance variance components are significantly different from 0 . If we fail to reject the null hypotheses, then we set the corresponding $\hat{\tau}$ values as 0 . If $\tau$ for a gene is significantly different from 0 , then we obtain its estimate using the EM algorithm described above. By applying this additional step, $E(\hat{\tau})$ would be closer to 0 when $\tau$ is 0 or close to 0 . When $\tau$ is relatively large, the conditional main effect test would reject $H_{0}: \tau=0$ and the final estimate is the same as the estimate from the original EM algorithm.

\section{Acknowledgements}

The authors thank the International Warfarin Pharmacogenetics Consortium and the PharmGKB resources for supplying the Warfarin data. They also thank Dr. Daowen Zhang for his helpful discussions and suggestions on the work. This work was supported by NIH grants R01 HG007508, R01 MH084022, and P01 CA142538.

\section{References}

1 Moore JH: The ubiquitous nature of epistasis in determining susceptibility to common human diseases. Hum Hered 2003;56:73-82.

12 Carlborg O, Haley CS: Epistasis: too often neglected in complex trait studies? Nat Rev Genet 2004;5:618-625.

-3 Greenspan RJ: The flexible genome. Nat Rev Genet 2001;2:383-387.

-4 Phenix H, Perkins T, Kærn M: Identifiability and inference of pathway motifs by epistasis analysis. Chaos 2013;23:025103.

-5 Barkoulas M, van Zon JS, Milloz J, van Oudenaarden A, Félix MA: Robustness and epistasis in the C. elegans vulval signaling network revealed by pathway dosage modulation. Dev Cell 2013;24:64-75.

6 Marchini J, Donnelly P, Cardon LR: Genomewide strategies for detecting multiple loci that influence complex diseases. Nat Genet 2005; 37:413-417.

7 Evans DM, Marchini J, Morris AP, Cardon LR: Two-stage two-locus models in genomewide association. PLoS Genet 2006;2:e157.

$\checkmark 8$ Zuk O, Hechter E, Sunyaev SR, Lander ES: The mystery of missing heritability: genetic interactions create phantom heritability. Proc Natl Acad Sci USA 2012;109:1193-1198.

$\checkmark 9$ Ioannidis JP: Non-replication and inconsistency in the genome-wide association setting. Hum Hered 2007;64:203-213.

10 Greene CS, Penrod NM, Williams SM, Moore $\mathrm{JH}$ : Failure to replicate a genetic association may provide important clues about genetic architecture. PLoS One 2009;4:e5639.

-11 Lin X, Hamilton-Williams EE, Rainbow DB, Hunter KM, Dai YD, Cheung J, Peterson LB, Wicker LS, Sherman LA: Genetic interactions among Idd3, Idd5.1, Idd5.2, and Idd5.3 protective loci in the nonobese diabetic mouse model of type 1 diabetes. J Immunol 2013;7: 3109-3120.

Gene-Gene Interactions with SimReg
12 Pillai R, Waghulde H, Nie Y, Gopalakrishnan K, Kumarasamy S, Farms P, Garrett MR, Atanur SS, Maratou K, Aitman TJ, Joe B: Isolation and high-throughput sequencing of two closely linked epistatic hypertension susceptibility loci with a panel of bicongenic strains. Physiol Genomics 2013;45:729-736.

13 Koh-Tan HH, McBride MW, McClure JD, Beattie E, Young B, Dominiczak A, Graham D: Interaction between chromosome 2 and 3 regulates pulse pressure in the stroke-prone spontaneously hypertensive rat. Hypertension 2013;62:33-40.

14 Howson JM, Cooper JD, Smyth DJ, Walker NM, Stevens H, She JX, Eisenbarth GS, Rewers M, Todd JA, Akolkar B, Concannon P, Erlich HA, Julier C, Morahan G, Nerup J, Nierras C, Pociot F, Rich SS, Type 1 Diabetes Genetics Consortium: Evidence of gene-gene interaction and age-at-diagnosis effects in type 1 diabetes. Diabetes 2012;11:3012-3017.

15 Ziyab AH, Davies GA, Ewart S, Hopkin JM, Schauberger EM, Wills-Karp M, Holloway JW, Arshad SH, Zhang H, Karmaus W: Interactive effect of STAT6 and IL13 gene polymorphisms on eczema status: results from a longitudinal and a cross-sectional study. BMC Med Genet 2013;14:67.

-16 Ma DQ, Rabionet R, Konidari I, Jaworski J, Cukier HN, Wright HH, Abramson RK, Gilbert JR, Cuccaro ML, Pericak-Vance MA, Martin ER: Association and gene-gene interaction of SLC6A4 and ITGB3 in autism. Am J Med Genet B Neuropsychiatr Genet 2010; 153B:477-483.

17 Bush WS, McCauley JL, Dejager PL, Dudek SM, Hafler DA, Gibson RA, Matthews PM, Kappos L, Naegelin Y, Polman CH, Hauser SL, Oksenberg J, Haines JL, Ritchie MD: A knowledge-driven interaction analysis reveals potential neurodegenerative mechanism of multiple sclerosis susceptibility. Genes Immun 2011;12:335-340.
18 Rothman KJ, Greenland S, Walker AM: Concepts of interaction. Am J Epidemiol 1980; 112:467-470

19 Siemiatycki J, Thomas DC: Biological models and statistical interactions: an example from multistage carcinogenesis. Int J Epidemiol 1981;10:383-387.

20 Chapman J, Clayton D: Detecting association using epistatic information. Genet Epidemiol 2007:31:894-909.

21 Cordell HJ: Detecting gene-gene interactions that underlie human diseases. Nat Rev Genet 2009; 10:392-404.

22 Murcray CE, Lewinger JP, Gauderman JW: Gene-environment interaction in genomewide association studies. Am J Epidemiol 2009;169:219-226.

23 Verzilli CJ, Stallard N, Whittaker JC: Bayesian graphical models for genomewide association studies. Am J Hum Genet 2006;79:100-112.

24 Zhang Y, Liu JS: Bayesian inference of epistatic interactions in case-control studies. Nat Genet 2007;39:1167-1173.

25 Kooperberg C, Ruczinski I: Identifying interacting SNPs using Monte Carlo logic regression. Genet Epidemiol 2005;28:157-170.

26 Zhang H, Bonney G: Use of classification trees for association studies. Genet Epidemiol 2000;19:323-332.

27 Sherriff A, Ott J: Applications of neural networks for gene finding. Adv Genet 2001;42: 287-297.

28 Lunetta KL, Hayward LB, Segal J, Van Eerdewegh P: Screening large-scale association study data: exploiting interactions using random forests. BMC Genet 2004;5:32.

29 Schwarz DF, König IR, Ziegler A: On safari to Random Jungle: a fast implementation of Random Forests for high-dimensional data. Bioinformatics 2011;27:439.

30 Jorgenson E, Witte JS: A gene-centric approach to genome-wide association studies. Nat Rev Genet 2006;7:885-891. 
31 Neale BM, Sham PC: The future of association studies: gene-based analysis and replication. Am J Hum Genet 2004;75:353-362.

- 32 Wang T, Ho G, Ye K, Strickler H, Elston RC: A partial least-square approach for modeling gene-gene and gene-environment interactions when multiple markers are genotyped. Genet Epidemiol 2009;33:6-15.

33 Chatterjee N, Kalaylioglu Z, Moslehi R, Peters U, Wacholder S: Powerful multilocus tests of genetic association in the presence of genegene and gene-environment interactions. Am J Hum Genet 2006;79:1002-1016.

34 Tzeng JY, Zhang D, Chang SM, Thomas DC, Davidian M: Gene-trait similarity regression for multimarker-based association analysis. Biometrics 2009;65:822-832.

35 Haseman JK, Elston RC: The investigation of linkage between a quantitative trait and a marker locus. Behav Genet 1972;2:3-19.

-36 Elston RC, Buxbaum S, Jacobs KB, Olson JM: Haseman and Elston revisited. Genet Epidemiol 2000;19:1-17.

- 37 Beckmann L, Fischer C, Obreiter M, Rabes M, Chang-Claude J: Haplotype-sharing analysis using Mantel statistics for combined genetic effects. BMC Genet 2005;6(suppl 1):S70.

- 38 Tzeng JY, Devlin B, Wasserman L, Roeder K: On the identification of disease mutations by the analysis of haplotype similarity and goodness of fit. Am J Hum Genet 2003;72:891-902.

-39 Wysowski D, Nourjah P, Swartz L: Bleeding complications with warfarin use: a prevalent adverse effect resulting in regulatory action. Arch Intern Med 2007;167:1414-1419.

-40 Wessel J, Schork NJ: Generalized genomic distance-based regression methodology for multilocus association analysis. Am J Hum Genet 2006;79:792-806.

41 Kwee LC, Liu D, Lin X, Ghosh D, Epstein MP: A powerful and flexible multilocus association test for quantitative traits. Am J Hum Genet 2008;82:386-397.

42 Schaid DJ: Genomic similarity and kernel methods I: advancements by building on mathematical and statistical foundations. Hum Hered 2010;70:109-131.
43 Price AL, Kryukov GV, de Bakker PIW, Purcell SM, Staples J, Wei LJ, Sunyaev SR: Pooled association tests for rare variants in exon-resequenced studies. Am J Hum Genet 2010;86: 832-838.

44 Tzeng JY, Zhang D, Pongpanich M, Smith C, McCarthy MI, Sale MM, Bradford BW, Hsu FC, Thomas DC, Sullivan PF: Detecting gene and gene-environment effects of common and uncommon variants on quantitative traits: a marker-set approach using gene-trait similarity regression. Am J Hum Genet 2011; 89:277-288.

45 Pongpanich M, Neely M, Tzeng JY: On the aggregation of multimarker information for marker-set and sequencing data analysis: genotype collapsing versus similarity collapsing. Front Genet 2012;2:110.

46 Duchesne P, Micheaux PL: Computing the distribution of quadratic forms: further comparisons between the Liu-Tang-Zhang approximation and exact methods. Comput Stat Data Anal 2010;54:858-862.

47 Kraft P, Yen YC, Stram DO, Morrison J, Gauderman W: Exploiting gene-environment interaction to detect genetic associations. Hum Hered 2007;63:111-119.

48 The International Warfarin Pharmacogenetics Consortium, Klein TE, Altman RB, Eriksson N, Gage BF, Kimmel SE, Lee MT, Limdi NA, Page D, Roden DM, Wagner MJ, Caldwell $\mathrm{MD}$, Johnson JA: Estimation of the warfarin dose with clinical and pharmacogenetic data. N Engl J Med 2009;360:753-764.

49 Yin T, Miyata T: Warfarin dose and the pharmacogenomics of CYP2C9 and VKORC1 rationale and perspectives. Thromb Res 2007; 120:1-10.

50 Puehringer H, Loreth RM, Klose G, Schreyer B, Krugluger W, Schneider B, Oberkanins C: VKORC1 $-1639 \mathrm{G}>\mathrm{A}$ and CYP2C9*3 are the major genetic predictors of phenprocoumon dose requirement. Eur J Clin Pharmacol 2010; 66:591-598.

51 Stehle S, Kirchheiner J, Lazar A, Fuhr U: Pharmacogenetics of oral anticoagulants: a basis for dose individualization. Clin Pharmacokinet 2008;47:565-594.

-52 Schalekamp T, Brassé BP, Roijers JF, van Meegen E, van der Meer FJ, van Wijk EM, Egberts AC, de Boer A: VKORC1 and CYP2C9 genotypes and phenprocoumon anticoagulation status: interaction between both genotypes affects dose requirement. Clin Pharmacol Ther 2007;81:185-193.
53 Schalekamp T, Brassé BP, Roijers JF, Chahid Y, van Geest-Daalderop JH, de Vries-Goldschmeding $\mathrm{H}$, van Wijk EM, Egberts AC, de Boer A: VKORC1 and CYP2C9 genotypes and acenocoumarol anticoagulation status: interaction between both genotypes affects overanticoagulation. Clin Pharmacol Ther 2006;80:13-22.

54 Bodin L, Verstuyft C, Tregouet DA, Robert A, Dubert L, Funck-Brentano C, Jaillon P, Beaune P, Laurent-Puig P, Becquemont L, Loriot MA: Cytochrome P450 2C9 (CYP2C9) and vitamin $\mathrm{K}$ epoxide reductase (VKORC1) genotypes as determinants of acenocoumarol sensitivity. Blood 2005;106:135-140.

55 van Schie RM, Babajeff AM, Schalekamp T, Wessels JA, le Cessie S, de Boer A, van der Meer FJ, van Meegen E, Verhoef TI, Rosendaal FR, Maitland-van der Zee AH, EUPACT study group: An evaluation of genegene interaction between the CYP2C9 and VKORC1 genotypes affecting the anticoagulant effect of phenprocoumon and acenocoumarol. J Thromb Haemost 2012;10:767-772.

56 Cerezo-Manchado JJ, Rosafalco M, Antón AI, Pérez-Andreu V, Garcia-Barberá N, Martinez AB, Corral J, Vicente V, González-Conejero $\mathrm{R}$, Roldán V: Creating a genotype-based dosing algorithm for acenocoumarol steady dose. Thromb Haemost 2013;109:146-153.

57 Nicolae DL, Gamazon E, Zhang W, Duan S, Dolan ME, Cox NJ: Trait-associated SNPs are more likely to be eQTLs: annotation to enhance discovery from GWAS. PLoS Genet 2010;6:e1000888.

58 Cooper GM, Shendure J: Needles in stacks of needles: finding disease-causal variants in a wealth of genomic data. Nat Rev Genet 2011; 12:628-640.

59 Buzkova P, Lumley T, Rice K: Permutation and parametric bootstrap tests for gene-gene and gene-environment interactions. Ann Hum Genet 2011;75:36-45.

-60 Wu MC, Maity A, Lee S, Simmons EM, Harmon QE, Lin X, Engel SM, Molldrem JJ, Armistead PM: Kernel machine SNP-set testing under multiple candidate kernels. Genet Epidemiol 2013;37:267-275 